\title{
Synthetic plasma edge diagnostics for EMC3-EIRENE, highlighted for Wendelstein 7- $\mathbf{X}^{\mathrm{a})}$
}

\author{
H. Frerichs, ${ }^{1, b)}$ F. Effenberg, ${ }^{1}$ O. Schmitz, ${ }^{1}$ C. Biedermann, ${ }^{2}$ Y. Feng, ${ }^{2}$ M. Jakubowski, ${ }^{2}$ R. König, ${ }^{2}$ M. \\ Krychowiak, ${ }^{2}$ J. Lore, ${ }^{3}$ H. Niemann, ${ }^{2}$ T. S. Pedersen, ${ }^{2}$ L. Stephey, ${ }^{4}$ and G. A. Wurden ${ }^{5}$ \\ 1) Department of Engineering Physics, University of Wisconsin - Madison, Madison, WI 53706, \\ $U S A$ \\ 2) Max-Planck-Institut für Plasma Physik, 17491 Greifswald, Germany \\ 3) Oak Ridge National Laboratory, Oak Ridge, TN 37831, USA \\ ${ }^{4)}$ HSX Plasma Laboratory, University of Wisconsin - Madison, Madison, WI 53706, \\ USA \\ ${ }^{5)}$ Los Alamos National Laboratory, Los Alamos, NM 87545, USA
}

(Dated: 23 June 2016)

\begin{abstract}
Interpretation of spectroscopic measurements in the edge region of high-temperature plasmas can be a challenge since line of sight integration effects make direct interpretation in terms of quantitative, local emission strengths often impossible. The EMC3-EIRENE code - a 3D fluid edge plasma and kinetic neutral gas transport code - is a suitable tool for full 3D reconstruction of such signals. A versatile synthetic diagnostic module has been developed recently which allows the realistic 3D setup of various plasma edge diagnostics to be captured. We highlight these capabilities with two examples for Wendelstein 7-X (W7-X): a visible camera for the analysis of recycling, and a coherent-imaging (CI) system for velocity measurements.
\end{abstract}

\section{INTRODUCTION}

The edge region of high-temperature plasmas is routinely analyzed with spectroscopic measurements. However, their interpretation in terms of local quantities can be challenging because of line of sight integration effects. Spatially resolved plasma data, on the other hand, is readily available from edge plasma modeling, which is performed for the Wendelstein 7-X (W7-X) stellarator with the EMC3-EIRENE code ${ }^{1,2}$. This code is applied by the fusion community to many devices throughout the world (see introduction and reference list in a recent publication on code improvements ${ }^{2}$ ). In combination with atomic data for photon emission, such plasma data allows for a full 3D reconstruction of signals from spectroscopic measurements.

A versatile synthetic diagnostic module is developed for the EMC3-EIRENE code that can facilitate diagnostic development as well as interpretation of experimental data. A brief introduction to the EMC3-EIRENE code and its derived data is given in section II since this provides the foundation for the synthetic edge plasma diagnostic. The framework of the synthetic diagnostic module is then described in section III, and its versatile capabilities are highlighted by two examples for W7-X: (1) a visible camera equipped with narrowband filters ${ }^{3,4}$ used for the analysis of recycling and impurity sources on the inboard limiter, and (2) a coherent-imaging (CI) system that is planned for implementation with the focus of velocity measurements.

\footnotetext{
a) Contributed paper published as part of the Proceedings of the 21st Topical Conference on High-Temperature Plasma Diagnostics, Madison, Wisconsin, June, 2016.

b) Electronic mail: hfrerichs@wisc.edu
}

\section{THE PLASMA EDGE DATA}

The EMC3-EIRENE code package ${ }^{1,2}$ allows modeling of the plasma edge in non-axisymmetric configurations, which can be found in stellarators and also in tokamaks when resonant magnetic perturbations are applied. The EMC3 code solves a set of steady state balance equations for particles, parallel momentum and energy of the main plasma species, and includes a reduced model for trace impurities. Resulting quantities are the plasma and impurity densities $n_{i}=n_{e}$ and $n_{I}$, the Mach number $M=v_{\|} / c_{s}$ based on the parallel plasma velocity $v_{\|}$ and the local sound speed $c_{s}$, and the electron and ion temperatures $T_{e}$ and $T_{i}$, respectively. Classical transport (Braginskii) is assumed along magnetic field lines, and "anomalous" cross-field transport is taken into account by free user parameters. Neutral particle densities and the source terms in the balance equations for the plasma due to interactions with neutral particles (ionization, charge exchange) are calculated by the EIRENE code. Both codes apply Monte Carlo methods and operate on a 3D finite flux tube grid for fast field line reconstruction. Plasma and neutral gas data is provided in each cell as a result.

During its first operational phase (OP1.1), W7-X is operated with a set of 5 inboard graphite limiters ${ }^{5}$. Application of the synthetic diagnostic module will be demonstrated based on modeling for this configuration ${ }^{6}$. W7$\mathrm{X}$ has 5 stellarator symmetric field periods, which allows the toroidal size of the simulation domain to be reduced to one half-period of $\Delta \varphi_{\text {sim }}=36 \mathrm{deg}$. For the present example, we use a radial and poloidal mesh resolution of $150 \times 673$ cells. The toroidal mesh resolution is 10 for a block of $\Delta \varphi=2 \mathrm{deg}$ at the position of the limiter, and 30 in the complementary block of the simulation domain. Input parameters for the sim- 


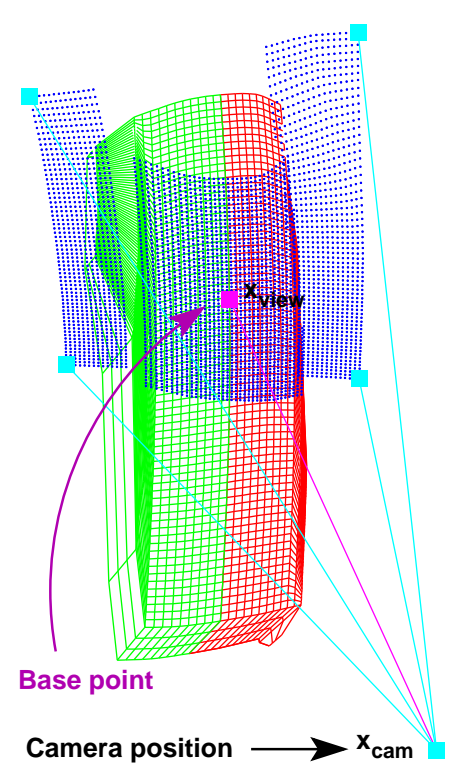

FIG. 1. Setup of a camera at the AEA30 port for observation of the inboard limiter. Observation points on the limiter and vessel are marked in blue. The transition between the green and red mesh marks the position of the up-down symmetric plane between field periods.

ulation are $n_{\text {sepx }}=1.3 \times 10^{13} \mathrm{~cm}^{-3}$ at the separatrix and $P_{\text {in }}=4 \mathrm{MW}$. Cross-field diffusion coefficients are set to $D_{\perp}=1 \mathrm{~m}^{2} \mathrm{~s}^{-1}$ and $\chi_{\perp}=3 D_{\perp}$. This example simulation is part of a parameter scan analysis ${ }^{6}$ ), and has been chosen only for the purpose of demonstrating the capabilities of the synthetic diagnostic module. More realistic parameters will be determined in a future analysis. Based on the simulation results, spatially resolved line emission data $\epsilon_{i \rightarrow j}$ is calculated from photon emissivity coefficients provided by OPEN-ADAS ${ }^{7}$ (derived class adf15), e.g. for $\mathrm{H}_{\alpha}$ emission from electron excitation:

$$
\epsilon_{H_{\alpha}}^{(\mathrm{exc})}=n_{e} \times n_{H} \times \mathrm{PEC}_{H_{\alpha}}^{(\mathrm{exc})}\left(n_{e}, T_{e}\right) .
$$

\section{THE SYNTHETIC DIAGNOSTIC MODULE}

The synthetic diagnostic is implemented in a generic way that allows easy configuration for different setups with only a few lines of input. The setup is defined by: 1. the camera position $\mathbf{x}_{\text {cam }}, 2$. the field of view and resolution, and 3 . the observable data. While an actual camera is limited to observe (filtered) line emission, the synthetic camera can observe the plasma itself, or any quantity $Q$ provided on the computational mesh. Each pixel of the synthetic camera image is given by the line of sight integral

$$
I=\int_{S} d s Q(s)=\sum_{k=1}^{n_{S}} \Delta s_{k} Q\left(i_{k}\right)
$$

a)
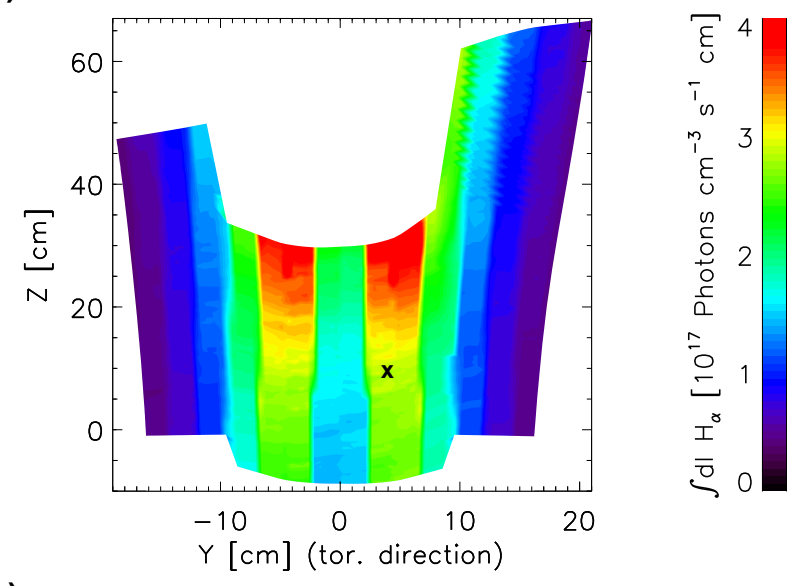

b)

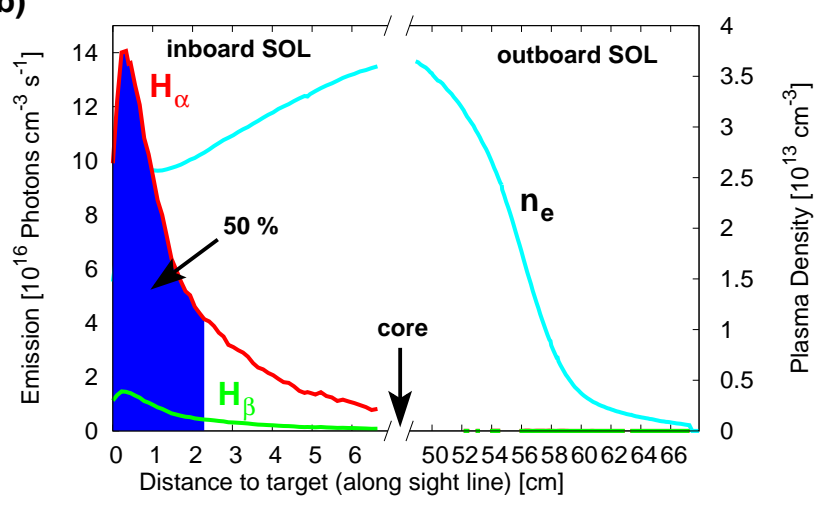

FIG. 2. (a) Synthetic camera image of the observable $\mathrm{H}_{\alpha}$ emission projected onto the limiter and vessel, (b) profiles along selected line of sight to point $\mathbf{x}$. $50 \%$ of the line of sight integrated $\mathrm{H}_{\alpha}$ emission originates in the blue area.

Since $Q$ is provided for each cell of the computational grid, the integral can be evaluated as the sum over all cell contributions $\Delta s_{k} Q\left(i_{k}\right)$ along the line of sight $\left(\Delta s_{k}\right.$ is length of the line of sight segment through cell $i_{k}$ ). While the default operation mode is to generate a synthetic camera image, it can also be used to generate line of sight profiles for selected pixels. This operation mode allows a spatially resolved analysis of the contributions to the integral observations. Example applications are presented below.

\section{A. Example: visible camera for direct limiter observation}

A visible camera observing one of the inboard limiters is operated at the AEA30 port in W7-X (see figure 1). The field of view is defined by a reference view point on the limiter $\mathbf{x}_{\text {view }}$, and an observation area of $36 \mathrm{~cm} \times$ $36 \mathrm{~cm}$ in a plane perpendicular to the base line of sight view $\mathbf{v}=\mathbf{x}_{\text {view }}-\mathbf{x}_{\text {cam }}$. The resolution is set to $64 \times 64$ pixels in this example, although it is straightforward to choose a higher resolution. The line of sight integrated 


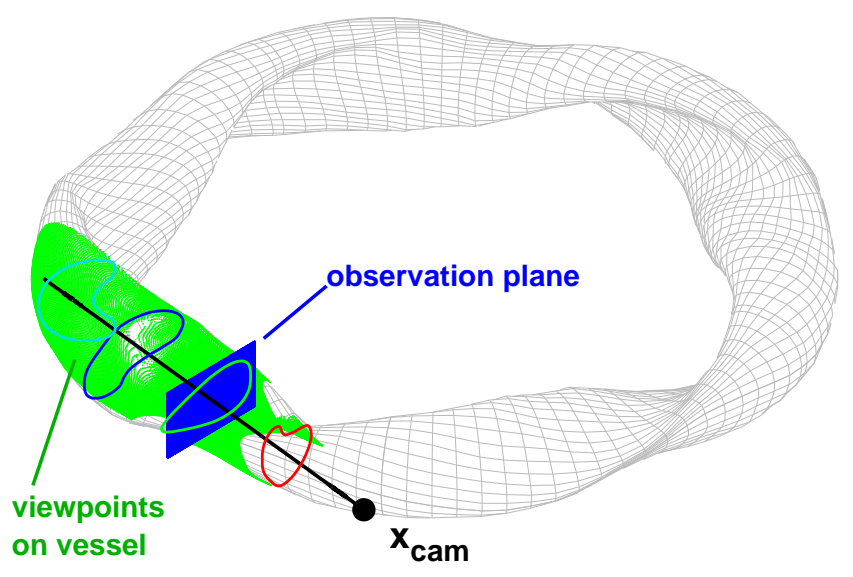

FIG. 3. Setup of a coherent-imaging system at and AEQ port. The observation plane is set at $\varphi=-36 \mathrm{deg}$ (blue), and the resulting view points on the vessel are indicated in green. A few cross-sections are highlighted for reference.

$\mathrm{H}_{\alpha}$ emission from this synthetic camera is shown in figure 2 (a) where coordinates have been mapped back from the observation plane (perpendicular to the base line of sight) onto the limiter and surrounding boundaries. A spatially resolved analysis for a selected line of sight is shown in figure 2 (b), which demonstrates that in the present plasma configuration $50 \%$ of the $\mathrm{H}_{\alpha}$ emission originates within $2.3 \mathrm{~cm}$ in front of the limiter.

\section{B. Example: coherent-imaging system for velocity measurements in the edge}

A second demonstration of the synthetic diagnostic is shown in figure 3 where the camera is set up with a toroidal view towards the $\varphi=-36 \mathrm{deg}$ plane. Velocity weighted line of sight integrals are calculated for evaluation of flow measurements by a coherent-imaging system. The resulting synthetic camera image based on CIII emission is shown in figure 4 . Such a setup is planned for implementation in W7-X for the next operation phase.

\section{CONCLUSIONS}

A synthetic diagnostic module has been developed for the EMC3-EIRENE code which can provide valuable information for the interpretation of spectroscopic measurements. The default operation mode is to generate a synthetic camera image, but it can also be used to generate line of sight profiles for selected pixels in order to analyze the spatial distribution of the contributions to the integral observations. Examples for two different setups have been presented: a visible camera observing the limiter during the first operational phase of $\mathrm{W} 7-\mathrm{X}$, and a coherent-imaging system to be implemented for velocity

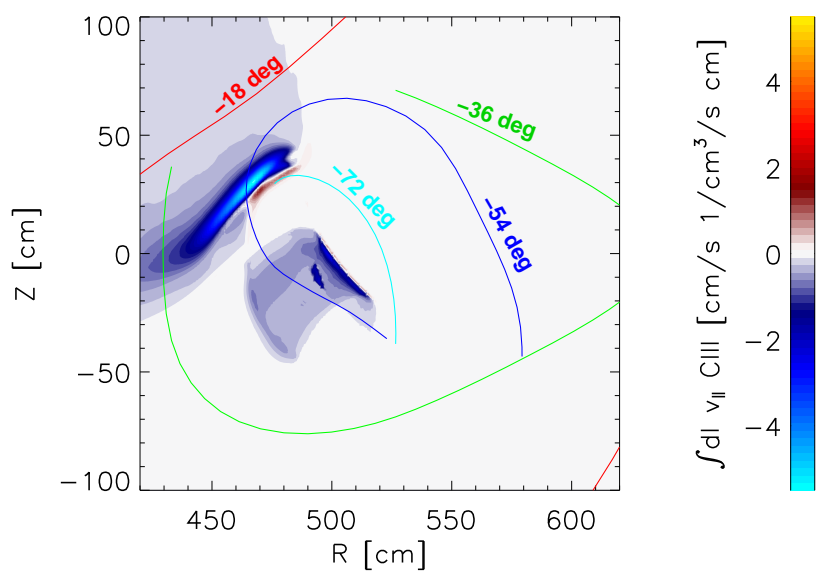

FIG. 4. Synthetic camera image of the velocity weighted CIII emission. The projected shape of a few toroidal slices are highlighted.

measurements at the plasma edge. The generic implementation of the synthetic diagnostic module makes the application to other configurations such as a helium beam

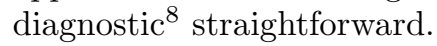

\section{ACKNOWLEDGMENTS}

This work was supported in part by the U.S. Department of Energy (DOE) under grant DE-SC0014210 and by startup funds of the Department of Engineering Physics at the university of Wisconsin - Madison. This work has been carried out within the framework of the EUROfusion Consortium and has received funding from the Euratom research and training programme 2014-2018 under grant agreement No 633053. The views and opinions expressed herein do not necessarily reflect those of the European Commission. The authors also wish to acknowledge the contributions and support of the W7-X team.

${ }^{1}$ Y. Feng, F. Sardei, and J. Kisslinger. 3D fluid modelling of the edge plasma by means of a Monte Carlo technique. Journal of Nuclear Materials, 266-269:812-818, 1999.

${ }^{2}$ Y. Feng et al. Recent Improvements in the EMC3-Eirene Code. Contrib. Plasma Phys., 54(4-6):426-431, 2014.

${ }^{3}$ L. Stephey et al. this conference. Rev Sci Intrum HTPD, 2016.

${ }^{4} \mathrm{G}$. A. Wurden et al. this conference. Rev Sci Intrum HTPD, 2016.

${ }^{5} \mathrm{~T}$. Sunn Pedersen et al. Plans for first plasma operation of Wendelstein 7-X. Nuclear Fusion, 55:126001, 2015.

${ }^{6}$ F. Effenberg et al. Numerical investigation of plasma edge transport and limiter heat fluxes in Wendelstein 7-X startup plasmas with EMC3-Eirene. Nuclear Fusion, (submitted to), 2016.

${ }^{7}$ OPEN-ADAS - Atomic Data and Analysis Structure: open.adas.ac.uk.

${ }^{8}$ T. Barbui et al. this conference. Rev Sci Intrum HTPD, 2016. 